\title{
Comparison of the Mason and KLM Equivalent Circuits for Piezoelectric Resonators in the Thickness Mode
}

\author{
Stewart Sherrit, Sean P. Leary, Benjamin P. Dolgin and Yoseph Bar-Cohen \\ Jet Propulsion Laboratory, California Institute of Technology, Pasadena, CA
}

\begin{abstract}
The parameters of the KLM and Mason's equivalent circuits in the thickness mode are presented to include dielectric, elastic and piezoelectric loss. The models are compared under various boundary conditions with and without acoustic layers to the analytical solutions of the wave equation. We show that in all cases equivalence is found between the analytical solution and the KLM and Mason's equivalent circuit models. It is noted that in order to maintain consistency with the linear equations of piezoelectricity and the wave equation care is required when applying complex coefficients to the models. The effect of the piezoelectric loss component on the power dissipated in the transducer is presented for loaded and unloaded transducers to determine the significance of the piezoelectric loss to transducer designers. The effect of the piezoelectric loss on the insertion loss was found to be small.
\end{abstract}

\section{INTRODUCTION}

Analytical solutions to the wave equation in piezoelectric materials can be quite cumbersome to derive from first principles in all but a few cases. Mason[1],[2] was able to show that for one-dimensional analysis most of the difficulties in deriving the solutions could be overcome by borrowing from network theory. He presented an exact equivalent circuit that separated the piezoelectric material into an electrical port and two acoustic ports through the use of an ideal electromechanical transformer as shown in Figure 1. The model has been widely used for free and mass loaded resonators[3], transient response[4], material constant determination[5], and a host of other applications[6]. One of the perceived problems with the model is that it required a negative capacitance at the electrical port. Although Redwood[4] showed that this capacitance could be transformed to the acoustic side of the transformer and treated like a length of the acoustic line it was still thought to be "un-physical". In an effort to remove circuit elements between the top of the transformer and the node of the acoustic transmission line Krimholtz, Leedom and Matthae[7] published an alternative equivalent circuit as shown in Figure 1. The model is commonly referred to as the KLM model and has been used extensively in the medical imaging community in an effort to design high frequency transducers [8],[9], multilayers[10], and arrays[11]. In the following sections we present the circuit parameter for the KLM and Mason's equivalent circuit for the case where the piezoelectric, dielectric and elastic constants are represented by complex quantities to account for intrinsic loss in the material.

\section{DESCRIPTION OF THE MODELS}

\section{General Description}

The KLM and Mason's model are shown in Figure 1 for the thickness mode. If the acoustic ports are shorted these models reduce to the free resonator equation derived from the linear piezoelectric equations and the wave equation [3] which has been adopted by the IEEE Standard on Piezoelectricity[12] for determination of the thickness material constants.

The constants of each model are shown in Table 1. In the KLM and Mason's equivalent circuit an electrical port is connected to the center node of the two acoustic ports representing the front and back face of the transducer. On the electrical port of the transformer all circuit elements are standard electrical elements and the voltage is related to the current via $\mathrm{V}=\mathrm{ZI}$ where $\mathrm{Z}$ is an electrical impedance. On the acoustical side of the transformer the force $F$ and the velocity $v$ are related through $F=\mathrm{Z}_{\mathrm{a}} v$ where $\mathrm{Z}_{\mathrm{a}}$ is the specific acoustic impedance $Z_{a} \propto \rho v A$ where $\rho$ is the density, $\mathrm{v}$ is the longitudinal velocity of the piezoelectric material and A is the area. It should be noted that the italic $v=\partial u / \partial t$ is a variable of the circuit model while the straight $\mathrm{v}$ is a constant of the material. The transformer is an ideal electromechanical transformer that conserves power during the transformation. The relationship between the constants of the free resonator and the KLM and Mason's equivalent circuits are shown in Table 1 in terms of the material constants of the free resonator. 


\section{Losses}

Although the material constants described in the IEEE Standards on Piezoelectricity are defined in terms of real coefficients a variety of authors (Holland [13], Berlincourt[14], Sittig [15], Katz[6], McSkimmin[16]) have suggested or used complex coefficients to describe the one or more loss components in many common piezoelectric materials.

Table 1. The complex material constants and the KLM and Masons parameters

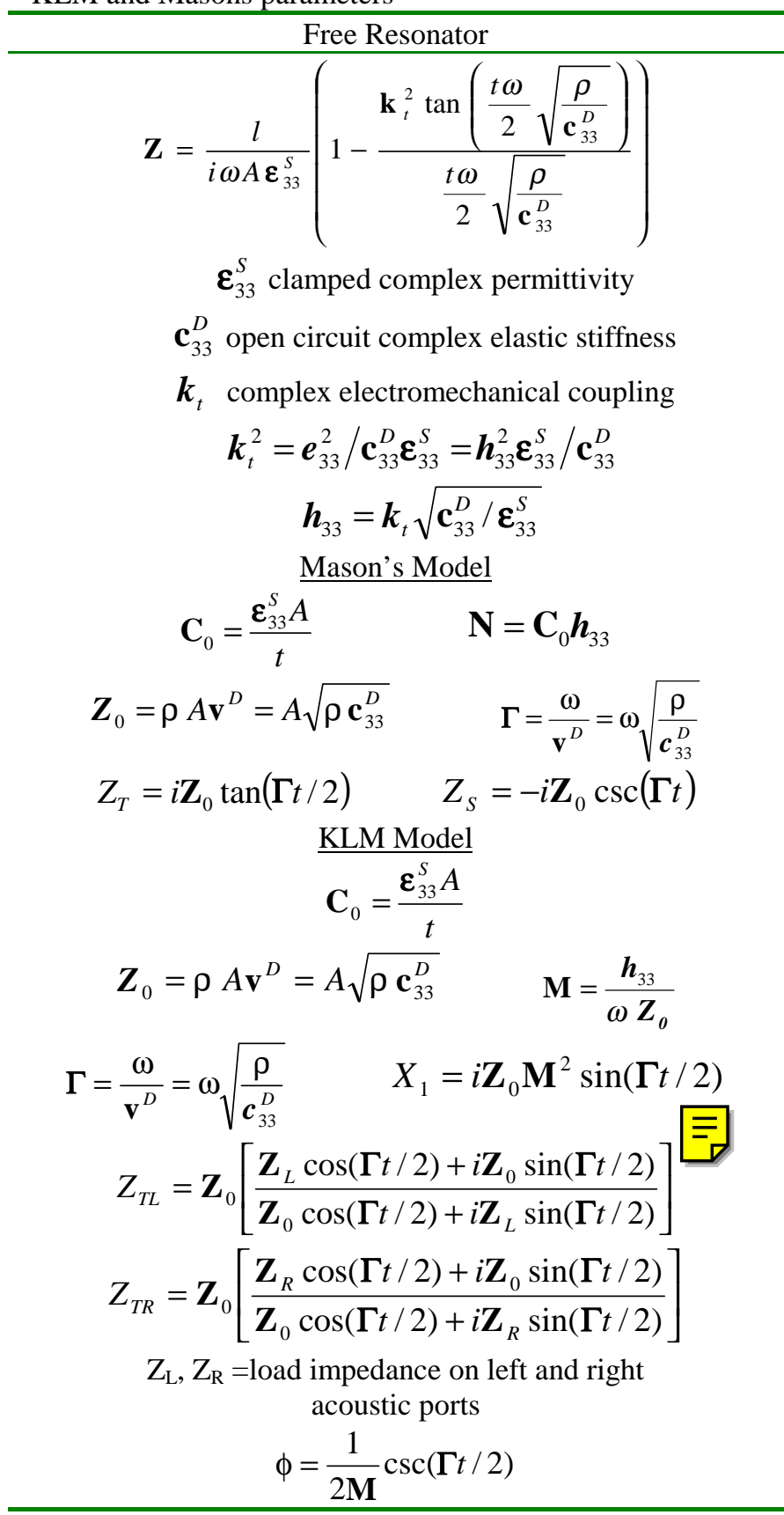

In the IEEE Standard of Piezoelectricity cautions are given about losses, dispersion, field, stress and time dependence (aging) of the material constants for linear piezoelectric materials. In the present study the material coefficients are defined as complex constants which assumes that the applied signals are small (extrinsic effects negligible), the sample is well aged and that we are in a normal rather than an anomalous dispersion regime where the frequency dependence of the material coefficients are nearly flat. Using the complex material constants the circuit parameters $\Gamma, \quad \mathrm{N}, \phi, \mathrm{Z}_{0}, \mathrm{M}, \mathrm{C}_{0}$ are now all treated as
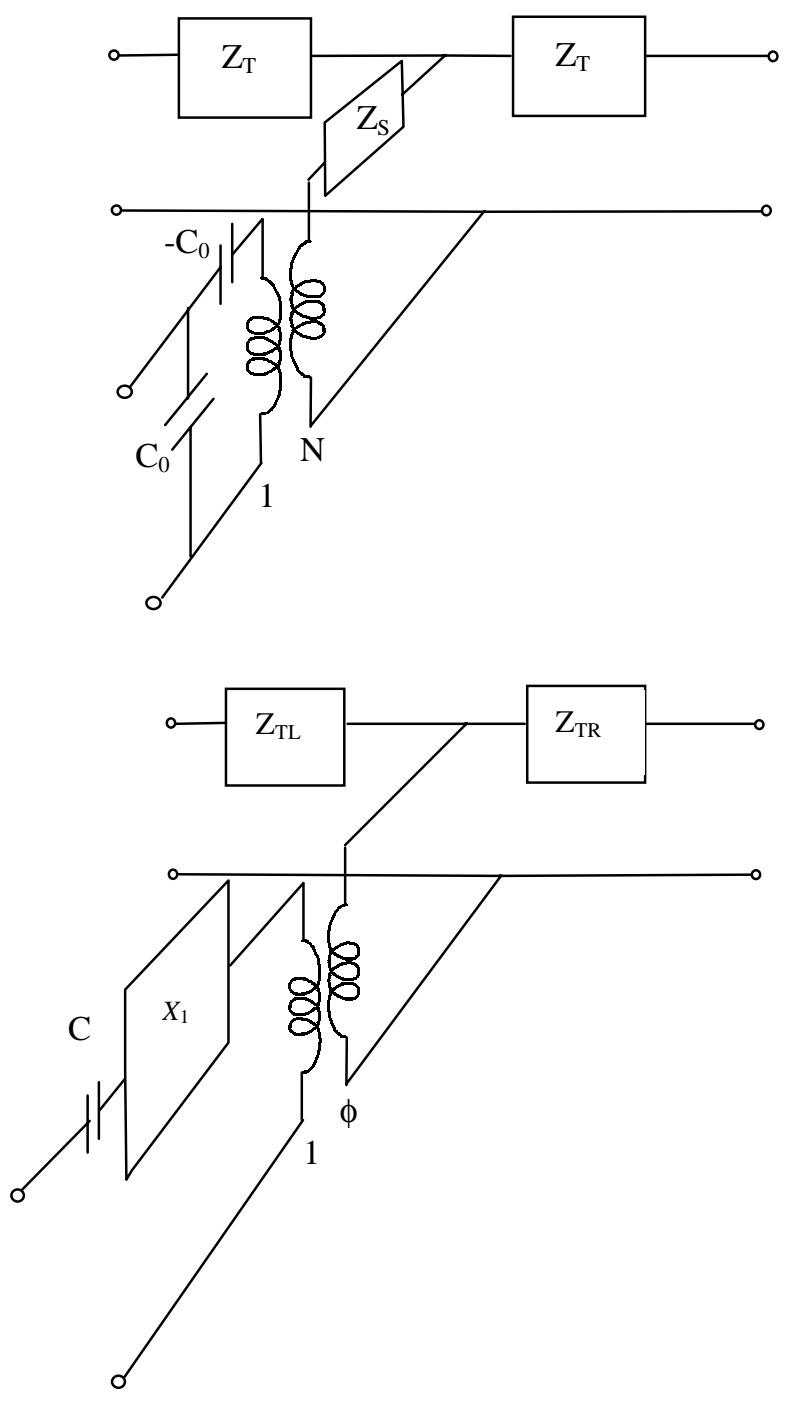

Figure 1. KLM and Mason's equivalent circuits. Quantities in figure are defined in Table 1.

complex quantities. Identities for trigonometric functions with complex arguments can be found by expanding the trigonometric function in exponential form. A list of these 
identities can be found in earlier work on equivalent lumped circuit constants of free piezoelectric resonators [17].

\section{Consistency in the application of loss}

It is worth noting that care must be taken when applying losses to the Mason and KLM equivalent circuits. In order to emphasize these points consider the case where the elastic and dielectric constants including losses have been determined from the resonance spectra of a free resonator and the electromechanical coupling is assumed to be real and determined from.

$$
k_{t}^{2}=\frac{\pi}{2} \frac{f_{S}}{f_{P}} \tan \left(\frac{\pi}{2} \frac{f_{P}-f_{S}}{f_{P}}\right)
$$

In this case it can be shown that the assumption of loss-less coupling actually implies from the equation for coupling defined in terms of the material constants

$$
k_{t}^{2}=\frac{e_{33}^{2}}{c_{33}^{D} \varepsilon_{33}^{S}}=\frac{h_{33}^{2} \varepsilon_{33}^{S}}{c_{33}^{D}}
$$

that a phase angle is present in each of the piezoelectric $\mathbf{e}_{33}$ and $\mathbf{h}_{33}$ coefficients (ie. these constants are complex). Rewriting the material constants including loss in polar form for this case $\left(\mathrm{k}_{\mathrm{t}}\right.$ real $)$ we find

$$
\begin{gathered}
\mathbf{e}_{33}=k_{t}\left|\mathbf{c}_{33}^{D}\right|^{1 / 2}\left|\boldsymbol{\varepsilon}_{33}^{S}\right|^{1 / 2} \exp i\left(\frac{\theta_{c^{D}}+\theta_{\varepsilon^{S}}}{2}\right) \\
\mathbf{h}_{33}=\frac{\left|\mathbf{e}_{33}\right|}{\left|\boldsymbol{\varepsilon}_{33}^{S}\right|} \exp i\left(\frac{\theta_{c^{D}}-\theta_{\varepsilon^{S}}}{2}\right)
\end{gathered}
$$

where $\theta_{c^{D}}$ and $\theta_{\varepsilon^{s}}$ are the phase angles associated with the loss components of the elastic stiffness and clamped permittivity. For example the stiffness phase angle is

$$
\theta_{c^{D}}=\arctan \left(\operatorname{Im}\left(c_{33}^{D}\right) / \operatorname{Re}\left(c_{33}^{D}\right)\right) \text {. }
$$

This means that in order to reproduce the fit to the data determined from the free resonator equation using either Mason's or the KLM equivalent circuit the piezoelectric constant in the equations for the turns ratio $\mathrm{N}$ (or the $\mathrm{M}$ coefficient for the KLM model) must be treated as complex as described in equations 3 and 4 . If these turns ratio coefficients are treated as real then it can be shown that for both the KLM and Mason's model we are assigning a non zero phase angle to $\mathrm{k}_{\mathrm{t}}$

$$
\begin{aligned}
& N_{\text {real }} \Rightarrow \theta_{k_{t}}=-\left(\theta_{\varepsilon^{s}}+\theta_{c^{D}}\right) / 2 \\
& h_{33 \text { real }} \Rightarrow \theta_{k_{t}}=\left(\theta_{\varepsilon^{s}}-\theta_{c^{D}}\right) / 2 \\
& M_{\text {real }} \Rightarrow \theta_{k_{t}}=\theta_{\varepsilon^{s}} / 2
\end{aligned}
$$

\section{MODELING}

\section{Open and Short Circuit Acoustic Ports}

The KLM and Mason's equivalent circuits were compared under half open, full open and short circuit conditions on the acoustic ports. The material constants (Motorola 3203HD) used for this comparison are shown in Table 2 along with the equivalent circuit parameters of each mode. Impedance data determine from the analytical solutions, and the KLM and Mason's equivalent circuit are shown in Figure 2. All data for each of the models is found to overlap.

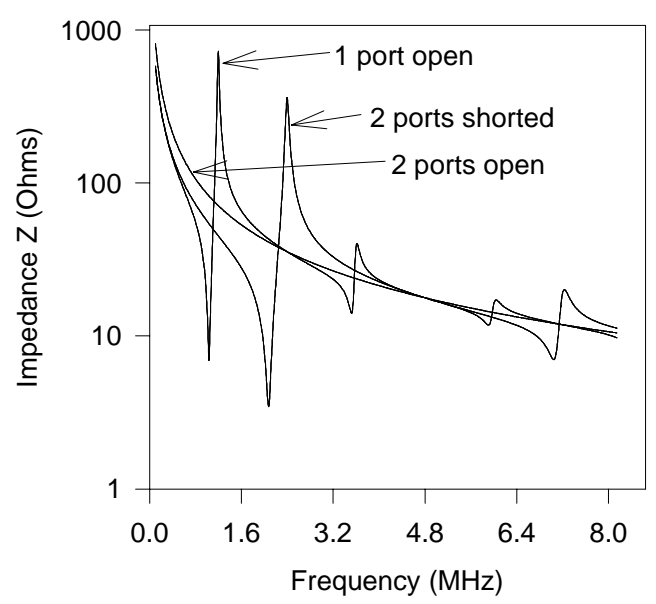

Figure 2. A comparison of the analytical solution, Mason's and the KLM model under various acoustic boundary conditions. All models were found to overlap.

\section{Layered Transducer Modeling}

The analytical solution for the impedance of a piezoelectric layer on a substrate was derived from the wave equation by Lakin, Kline and McCarron[18], A more recent derivation by Lukacs et al[19] extended the solution to include loss in the elastic, dielectric and piezoelectric constants and first order dispersion in the dielectric constant. These solutions are valid for all cases where the lateral dimensions of the acoustic layer and the piezoelectric layer are much larger than either layer thickness.

In the following section Mason's and KLM equivalent circuits are compared to the analytical solution for a high impedance backing (stainless steel) and a low 
impedance backing (epoxy) to investigate the effects of including an acoustic layers on the various models.

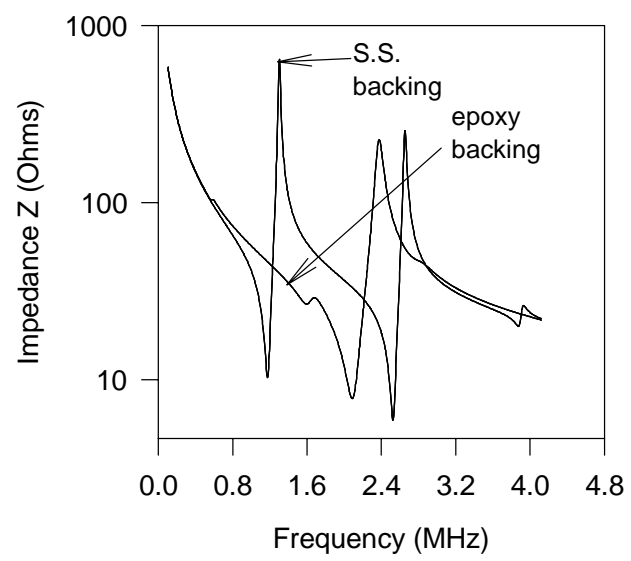

Figure 3. A comparison of the analytical solution, Mason's and the KLM model with stainless steel and epoxy backing layers. All models were found to overlap.

The geometry, stiffness, density and velocity, of the each of the backing layers are listed in Table 3. The piezoelectric properties for the Motorola 3203 HD material used in this simulation are shown in Table 2. The complex material properties were determined using Smits' method[20].

The models are similar to the models presented in Figure 1 however each model also has an acoustic transmission line element attached to one of the acoustic ports of the piezoelectric to represent the backing layer.

The results are shown in Figure 3. The analytical soloution, and the KLM and Mason's equivalent circuit were found to produce identical impedance curves if the loss was treated consistantly as discussed previously.

\section{B. Effect of Piezoelectric Loss on Power Spectrum}

The electrical power factor for the free resonator shown in Figure 2 calculated using the material constants in Table 2 is shown in Figure 4. The electrical power factors for the same resonator with $\mathrm{k}_{\mathrm{t}}$ real, $\mathrm{e}_{33}$ real and $\mathrm{h}_{33}$ real calculated using equation 2 are also shown in the figure. It is clear for the free resonator significant difference in the power spectra are apparent below, above and between $f_{\mathrm{S}}$ and $f_{\mathrm{P}}$. In the case where $\mathrm{k}_{\mathrm{t}}$ is assumed to be real, the power dissipated above and below resonance is symetric about the midpoint between $f_{\mathrm{S}}$ and $f_{\mathrm{P}}$. This means that one can assess the validity of the assumption of $\mathrm{k}_{\mathrm{t}}$ real by plotting the $\mathrm{R} / \mathrm{Z}$ data as a function of frequency and noting whether the dissipation is symetric about the midpoint between $f_{\mathrm{S}}$ and $f_{\mathrm{P}}$. In order to see the effect of the piezoelectric loss on a tuned transducer rather than a free resonator we have used Mason's equivalent circuit to model a backed $25 \mathrm{MHz}$ transducer radiating into water. The power dissipated in the piezoelectric element of the transducer and the 2 way insertion loss are shown in Figures 5 and 6 . The piezoelectric material properties are from Table 2. The dimensions of the piezoelectric were $0.1 \mathrm{~mm} \times 1 \mathrm{~mm}$ squared. The tuning inductor was $492 \mathrm{nH}$ 's. The source inpedance was $50 \Omega$. The specific acoustic impedance of water was $Z=\rho A v=6 \mathrm{~kg} / \mathrm{s}$. The density, length and velocity of the backing layer were $\rho_{b}=1700$ $\mathrm{kg} / \mathrm{m}^{3}, 1=0.002 \mathrm{~m}$ and $\mathrm{v}=2700(1+0.005 \mathrm{i})$.

Figure 4. Electrical power factor for the measured

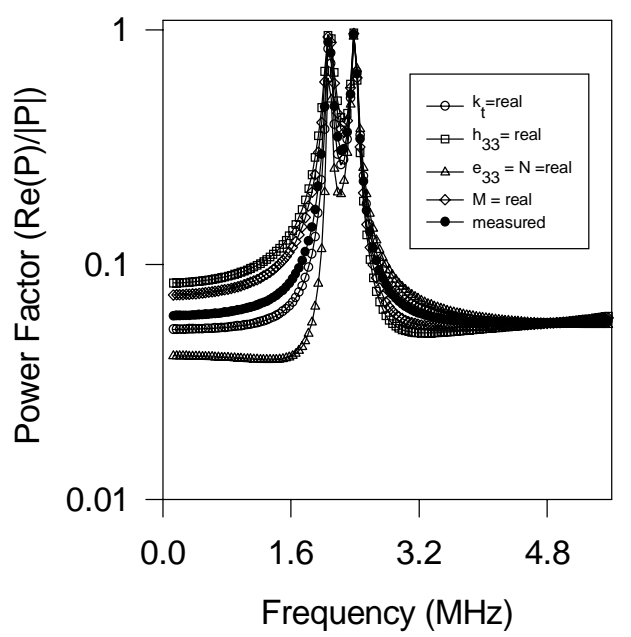

resonator and assuming lossless material constants

The power dissipated in the piezoelectric element is seen to have a signifigant dependence on the piezoelectric loss designation. This means that for $\mathrm{S} / \mathrm{N}$ calculations, duty cycling, and heating effects properly including the piezoelectric loss can aid in acurately determining these aspects of the transducer design.

The effect of the loss designation is not as apparent on the general shape of the insertion loss curve shown in Figure 6. However at $f_{\mathrm{S}}$ the curves deviate by about $3 \mathrm{~dB}$ from each other. It is clear given the other approximations (electrode mass, sheet resistivity, potting effects, mode coupling coupling, dispersion and parasitic impedances are all neglible) assumed in the modeling that 
the piezoelectric loss has very little effect on the overall shape and bandwith of the insertion loss.

Table 2. Complex material constants determined from a average of 5 samples. KLM and Masons parameters for Motorola 3203HD material.

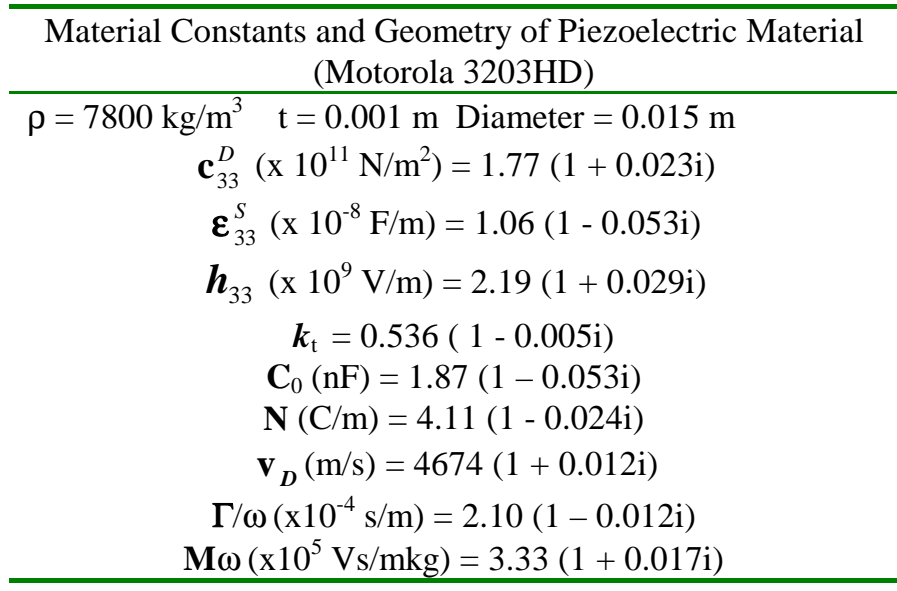

Table 3. The acoustic properties of the epoxy and stainless steel backing materials

\begin{tabular}{c}
\hline $\mathrm{t}=0.001 \mathrm{~m} \quad$ Diameter $=0.015 \mathrm{~m}$ \\
$\underline{\text { Epoxy }}$ \\
\hline$\left(\mathrm{kg} / \mathrm{m}^{3}\right)=1100$ \\
$\mathbf{c}_{33}^{D}\left(\mathrm{x} 10^{9} \mathrm{~N} / \mathrm{m}^{2}\right)=5.3(1+0.1 \mathrm{i})$ \\
$\mathbf{v}_{D}(\mathrm{~m} / \mathrm{s})=2200(1+0.05 \mathrm{i})$ \\
$\Gamma / \omega\left(\mathrm{x} 10^{-4} \mathrm{~s} / \mathrm{m}\right)=4.53(1-0.05 \mathrm{i})$ \\
Stainless Steel \\
$\rho\left(\mathrm{kg} / \mathrm{m}^{3}\right)=7890$ \\
$\mathbf{c}_{33}^{D}\left(\mathrm{x} 10^{11} \mathrm{~N} / \mathrm{m}^{2}\right)=2.645(1+0.002 \mathrm{i})$ \\
$\mathbf{v}_{D}(\mathrm{~m} / \mathrm{s})=5790(1+0.001 \mathrm{i})$ \\
$\Gamma / \omega\left(\mathrm{x} 10^{-4} \mathrm{~s} / \mathrm{m}^{2}\right)=1.727(1-0.001 \mathrm{i})$
\end{tabular}

It also should be noted that setting the dielectric and elastic losses to zero was found to have very little effect on the insertion loss curve. The general shape of the insertion loss was found to be primarly dependent on the source impedance, tunning inductor, acoustic impedance of the backing and the water and the real parts of the material coefficients. It is interesting to note that for the

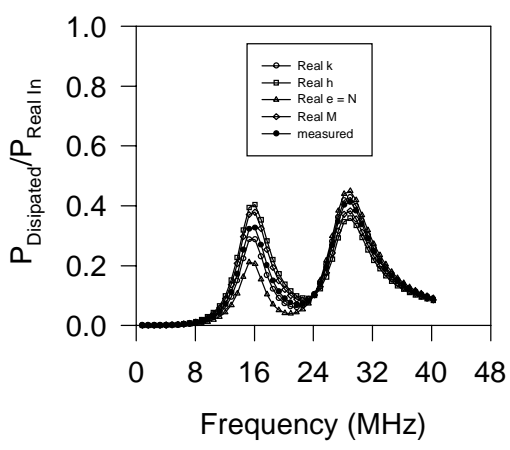

Figure 5. The electrical power dissipated in the piezoelectric element of the transducer described in text for $\mathrm{k}_{\mathrm{t}}$ measured, $\mathrm{k}_{\mathrm{t}}$ real, $\mathrm{e}_{33} \propto \mathrm{N}$ real, $\mathrm{h}_{33}$ real., and $\mathrm{M}$ real.

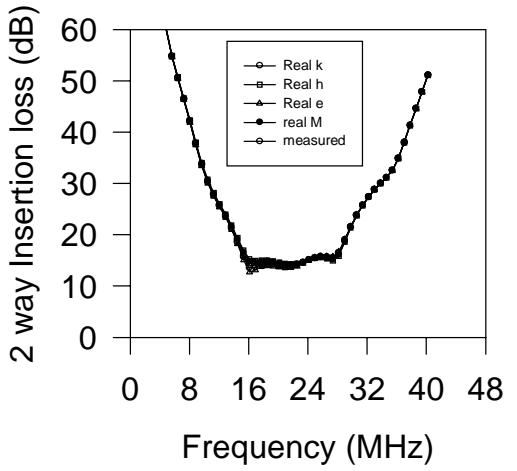

Figure 6. The insertion loss for the various piezoelectric loss designations.

Motorola 3203 HD material that larger errors in the modeling occur due to the inconsistancies in the application of the loss compared to the assumption that $\mathrm{k}_{\mathrm{t}}$ is a real number.

\section{CONCLUSIONS}

The parameters of the KLM and Mason's equivalent circuits in the thickness mode were presented for the case where dielectric, elastic and piezoelectric losses are present. The models were compared and found to be equivalent under a variety of boundary conditions when the loss was applied consistently in each of the models. The effect of piezoelectric, dielectric and elastic loss coefficients on the 
overall shape of the insertion loss curve was found to be small and independent of the designation of piezoelectric loss. The dissipated power in the transducer was found to be dependent on the piezoelectric loss designation, which suggested that the piezoelectric loss is important for determining heating effects in the transducer.

\section{ACKNOWLEDGMENT}

The research at the Jet Propulsion Laboratory (JPL), a division of the California Institute of Technology, was carried out under a contract with the National Aeronautics Space Agency (NASA).

\section{REFERENCES}

[1] W..P. Mason, Electromechanical Transducers and Wave Filters, Princeton, NJ, Van Nostrand, 1948

[2] W.P. Mason, Physical Acoustics and the Properties of Solids, D. Van Nostrand Co., Princeton, NJ, 1958

[3]D. A. Berlincourt, D.R. Curran, H. Jaffe, "Chapter 3Piezoelectric and Piezomagnetic Material and their Function in Transducers, pp. 169-270, Physical AcousticsPrinciples and Methods, Volume 1-Part A, ed. W.P. Mason Academic Press, New York, 1964

[4] M. Redwood, "Transient Performance of a Piezoelectric Transducer", Journal of the Acoustical Society of America, 33, pp.527-536, 1961

[5] S. Saitoh, H Honda, N. Kaneko, M. Izumi, S. Suzuki, The Method of Determining $\mathrm{k}_{\mathrm{t}}$ and $\mathrm{Q}_{\mathrm{m}}$ for low $\mathrm{Q}$ piezoelectric materials" Proceedings of the IEEE Symposium on Ultrasonics, San Francisco, CA, pp. 620623,1985

[6] Solid State Magnetic and Dielectric Devices, edited by H.W. Katz, John Wiley and Sons, New York, 1959

[7] R. Krimholtz, D.A. Leedom, G.L. Matthaei, "New Equivalent Circuit for Elementary Piezoelectric Transducers", Electron Letters, Electron. Lett. 6, pp. 398399,1970

[8] M. J. Zipparo, K.K. Shung, T.S. Shrout, "Piezoceramics for High Frequency (20-100 MHz) Single Element Imaging Transducers", IEEE Trans on Ultrasonics, Ferroelectrics and Frequency Control, 44, pp.1038-1048, 1997

[9] F.S. Foster, L.K. Ryan, D.H. Turnbull, "Characterization of Lead Zirconate Titanate Ceramics for Use in Miniature High Frequency $(20-80 \mathrm{MHz})$ Transducers, IEEE Trans on Ultrasonics, Ferroelectrics and Frequency Control , 38, pp. 446-453, 1991

[10] Q. Zhang, P.A. Lewin, P.E. Bloomfield, "PVDF Transducers- A Performance Comparison of Single-Layer and Multilayer Structures", IEEE Trans on Ultrasonics, Ferroelectrics and Frequency Control, 44, pp.1148-1156, 1997
[11] R.L. Goldberg, M.J. Jurgens, D.M. Mills, C.S. Henriquez, D.Vaughan, S. W. Smith, "Modeling of Piezoelectric Multilayer Ceramics Using Finite Element Analysis", IEEE Trans on Ultrasonics, Ferroelectrics and Frequency Control., 44, pp. 1204-1214, 1997

[12] IEEE Standard on Piezoelectricity, IEEE/ANSI Std. 176-1987

[13]R. Holland, IEEE Transactions on Sonics and Ultrasonics, " Representations of the Dielectric, Elastic, and Piezoelectric Losses by Complex Coefficients", IEEE Transactions on Sonics and Ultrasonics, SU-14, pp.18-20, 1967

[14] D. A. Berlincourt, Chapter 2 -"Piezoelectric Crystals and Ceramics", Ultrasonic Transducer Materials, edited by O.E. Mattiat, Plenum Press, New York, 1971

[15]E.K. Sittig, Chapter 5-, "Design and Technology of Piezoelectric Transducers for Frequencies above 100 MHz", Physical Acoustics, Vol IX, edited by W.P. Mason, R.N. Thurston, Academic Press, New York, 1972

[16]H.J. McSkimmin , "Chapter 4- Ultrasonic Methods for Measuring the Mechanical Properties of Liquids and Solids, pp. 271-334, Physical Acoustics-Principles and Methods, Volume 1-Part A, ed. W.P. Mason Academic Press, New York, 1964

[17] S. Sherrit, H.D. Wiederick, B.K. Mukherjee, M. Sayer, "An accurate equivalent circuit for the unloaded piezoelectric vibrator in the thickness modes." J. Phys. D. (Applied Physics), 30, pp. 2354-2363, 1997

[18] Kenneth M. Lakin, Gerald R. Kline, Kevin T. McCarron, "High Q Microwave Acoustic Resonators and Filters" IEEE Trans. On Microwave Theory and Techniques, 41, pp. 2139-2146, 1993

[19] M. Lukacs, T. Olding, M. Sayer, R. Tasker, S. Sherrit, "Thickness mode material constants of a supported piezoelectric film", J. of Applied Physics , 85, pp.28352843, 1999

[20] J.G. Smits," Iterative Method for the Accurate Determination of the Real and Imaginary Material Coefficients of Piezoelectric Ceramics", IEEE Trans on Sonics and Ultrasonics, SU-23, pp. 393-402, 1976 